\title{
Analysis on the Coordination Problem of Backup Automatic Switch and Voltage Juxtaposition device In Special Mode
}

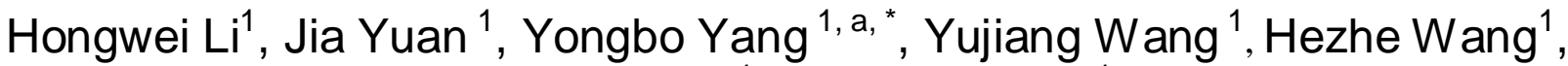 \\ Xiaodong $\mathrm{Gu}^{1}$ and Dan Zhang ${ }^{1}$ \\ ${ }^{1}$ State Grid Zhengzhou Power Supply Company Zhengzhou, Henan 450052 \\ a, ${ }^{*} 18625567505 @ 163 . c 0 m$
}

Keywords: Backup Automatic Switch; Voltage juxtaposition device; VT

\begin{abstract}
This paper briefly introduces the specific meaning of the backup automatic switch and voltage juxtaposition device and their application, and analyzes the problems of the Backup Automatic Switch and Voltage juxtaposition device in different primary wiring schemes. Three different principles are put forward to improve the program. The similar situation in the power grid with the VT juxtaposition device with the work of the relevant work provides a basis.
\end{abstract}

\section{Introduction}

The function of the backup automatic switch is that the power supply can be automatically put into operation when the power system is faulty or other causes the working power source is disconnected, or the power supply is automatically put into other normal working power. This is to improve the reliability of power supply, to ensure continuity of power supply an effective means.

Voltage transformers (TV) as an important primary device in the power system plays a vital role. When the backup automatic switch automatically input, it is a key that the voltage transformer secondary circuit can switch properly. If there is a problem in this process, it will bring serious problems to the whole secondary system, and it is very important to ensure the stable operation of the voltage transformer and its secondary circuit.

At present, the internal bridge connection in $110 \mathrm{kV}$ substation applications more and more widely, but also make the logic of self-cast has become quite complex, to bring some difficulties to operation and maintenance. This paper discusses a special internal bridge connection mode, the preparation of self-cast voltage transformer secondary side of the problem, and summed up the corresponding preventive measures.

\section{The Backup Automatic Switch and Voltage Juxtaposition Device}

\section{Backup Automatic Switch}

There are some different modes of the backup automatic switch, as the bus-coupler or bridge from the cast, into the line from the cast, the transformer from the cast and special circumstances, such as with a small power supply, balanced load from the vote. In this paper, a brief analysis of the bridge connection.

The action logic of the mother or bridge self-cast is that after satisfying all the operating conditions of the self-cast, the self-cast device sends out the trip exit command after the delay. Since bus I with no pressure and no flow, and to meet the section that bus II with pressure, by the time delay the circuit breaker without voltage tripping. After confirming the voltage-loss circuit breaker tripping delay, close the bus-bar or bridge circuit breaker.

\section{Voltage Juxtaposition}

In the design process of power engineering, application of voltage transformer, voltage are often encountered problems. The voltage juxtaposition is that when a section of one bus voltage transformer is out of operation, and when the bridge switch is in the operating position, due to the protection of the 
measurement and other reasons, it is necessary to use the operating voltage transformer to supply the two bus voltage. When the two sections of the bus need to run in parallel, usually in the electric equipment installed on the voltage parallel device. Voltage juxtaposition device is commonly used in the single bus-bar connection mode, the double bus wiring or internal bridge connection.

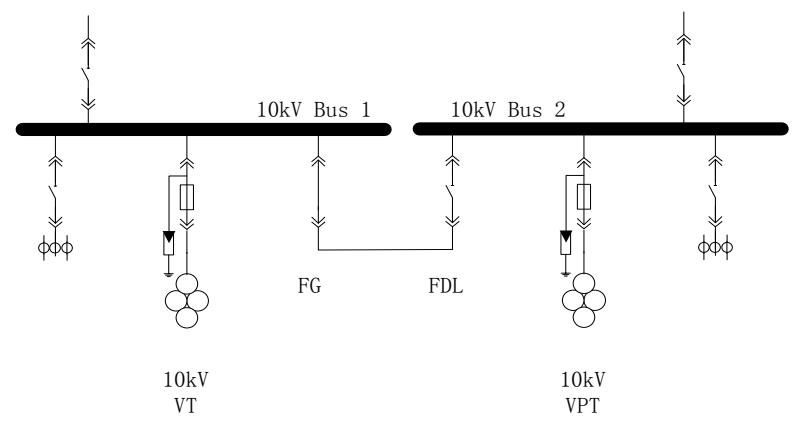

Figure 1. Mode of single bus-bar with segments connection

In the case of single parent segment connection, when the isolated handcart and the circuit breaker are in the operating position, the two section bus is connected. If you need to withdraw from the operation of a VT, just start the voltage juxtaposition device, so that the juxtaposition relay is on, and then the VT can be removed from the workplace. This is the whole working process of the VT device.

\section{The Coordination of Backup Automatic Switch and Voltage Juxtaposition Device}

Taking the Backup Automatic Switch device of single bus-bar with segments connection as an example. Normal operation, the two transformers with a bus running, the two section of the bus into the main circuit breaker 1DL, 2DL is in place, the sectional breaker FDL in the spare parts, 1PT, 2PT as the bus PT. When the upper power failure caused the loss of pressure on bus I, Backup Automatic Switch act to jump 1DL, close FDL, all load supply by the power transformer 2 . At the same time, the isolated handcart and the sectional circuit breaker are in the operating position, that is, the two sections of the bus are running in parallel. The voltage juxtaposition device satisfies the parallel condition, and the two voltage circuit is automatically tied.

\section{The Coordination Problem under Special Connection Mode}

However, there are some problems in the coordination of the Backup Automatic Switch device and the Voltage juxtaposition device in the special connection mode. When the device has been put into operation, the two voltage can not be properly coordinated. Even in some cases, the coordination of the voltage may lead to an accident.

Here we focus on the analysis of the corresponding circuit in Figure 3, the work process of voltage juxtaposition device. As shown in Figure 3, the voltage transformer can be used as a line voltage transformer before the circuit breaker is switched on, and it can provide the voltage of the input line for the Backup Automatic Switch device. After the closing, the circuit breaker as the bus voltage transformer, the voltage can provide the bus voltage for the protection device, the measurement and control device and the metering device. The voltage juxtaposition device which is suitable for the primary connection and that used for a single bus section or a double bus bar have certain differences on the wiring. 


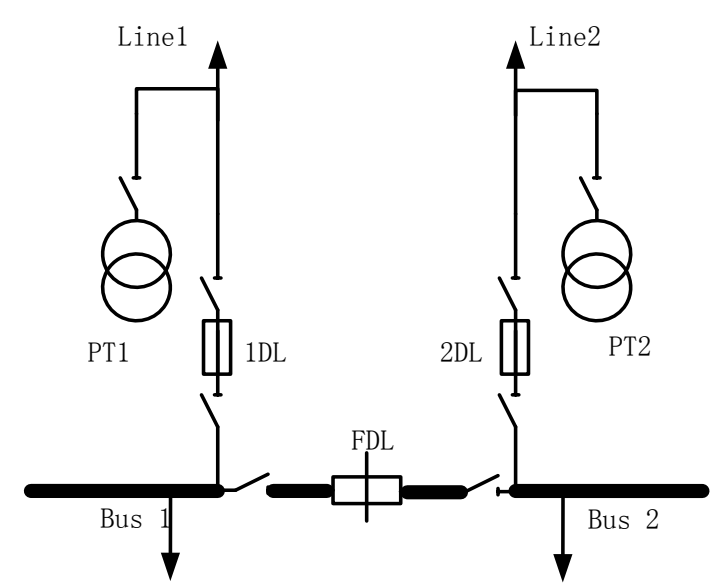

Figure 2. Mode of a special type of bridge connection

A case study on the Backup Automatic Switch of bridge switch. Normal operation, the 2 lines running with 1DL, 2DL in the operating position, 1PT, 2PT as the bus PT. When the line 1 failure, usually excising the failure by the opposite side of the protection, at this time, for the preparation of the Backup Automatic Switch device, the bus 1 of the loss of pressure, the bus 2 with a pressure, to jump 1DL, and close FDL, thus transformer 1 is supplied by line 2. The pressure loss of the protection of the transformer, the measuring device and the measuring device, So you need to start the voltage juxtaposition device. Usually, this process should be completed automatically to ensure that the transformer protection, measurement and control devices, measuring devices to restore the voltage quickly.

Since there is no closing contact control of bridge breaker, in this case the 1PT isolation switch is still in the operating position, after the secondary voltage combines, a reversed feeding fault from secondary to primary circuit though the voltage juxtaposition device. Obviously, if the two wiring of the device is consistent with the usual internal bridge connection, it is unreasonable.

After the voltage is sent back to the primary device, if there is no security on the side of the line, someone will contact the line, which will cause electric shock injury.

\section{The Improvement of the Special Connection}

In view of the different wiring way, this kind of phenomenon should be avoided in the design of the circuit. In fact, the query related protection scheme, automatic voltage juxtaposition device principle, we did not find the targeted measures. As the equipment supplier, not pay enough attention to this problem, in the device design and terminal design, there is no effective interface provided on this scheme.

Based on the analysis of the principle of voltage juxtaposition device, this paper puts forward the following three kinds of solutions according to the special internal bridge connection mode, which includes both line voltage transformer and bus voltage transformer function.

\section{Quit the Fault Line Voltage Transformer}

In order to prevent the secondary voltage back to primary circuit, the most intuitive way is to be forced to stop the line of the voltage transformer out of operation.

In this way, the voltage juxtaposition device set in manual processing mode. The practical production process, before starting voltage juxtaposition device, the fault line voltage transformer will be out of operation by the substation operator. This method is not practical and feasible in practice. First of all, in the current developing mode, the mode of operation and maintenance of the substation has been changed from the previous multi-duty to fewer people on duty or unattended transformation, in some areas has been achieved unattended substation mode. In this way, the line fault, the operation and maintenance of substation personnel can not guarantee the timely delivery of the voltage 
transformer. Even if the operator in the field to carry out maintenance work, exit the fault VT also takes time, it will mean that the transformer protection, measurement and control devices, metering devices for a long period of time, the loss of voltage.

In another way, the voltage parallel device is arranged in the automatic parallel mode. When the device is put into operation, the remote operation is carried out by the monitor, and the voltage transformer is exited. This requires the isolation switch is also to meet the "remote control" conditions, obviously, to achieve this feature requires a considerable period of time, as well as a huge economic investment, so this program is not easy.

\section{Increase the Export of Backup Automatic Switch Device}

When the line fault and backup automatic switch device is operated, the circuit of the backup automatic switch device is added, and the fault line VT is pulled out. This scheme can solve the problem, but put forward more requirements for the output circuit of the backup automatic switch device, and requires the use of electric operating mechanism of the VT circuit isolation switch.

If we consider this problem in the early stage of design, we can increase the output circuit to meet the requirements, but if the existing automatic input device is not enough, it is difficult to realize this function. In view of the substation has been completed the technological transformation project, it requires a lot from the new wiring, tripping circuit consists of two lines spaced voltage transformer isolation switch, and the voltage juxtaposition device needs to exit a long time, and two lines in outage to meet the technical demand. The program is more complicated.

At the same time, this kind of internal bridge connection line in the side of the rapid grounding locking logic, the corresponding line of the VT isolating switch gate, and meet the conditions of the line without pressure. In the accident treatment, it is necessary to restore the VT device again, prolong the time of accident disposal.

\section{Increase the Contact Position of the Circuit Breaker}

In addition, the secondary connection of the voltage juxtaposition device is improved, and the auxiliary contacts of two incoming circuit breakers are added in the two connection circuit of the voltage juxtaposition device. The secondary connection of the voltage juxtaposition device can be improved according to figure 3 .

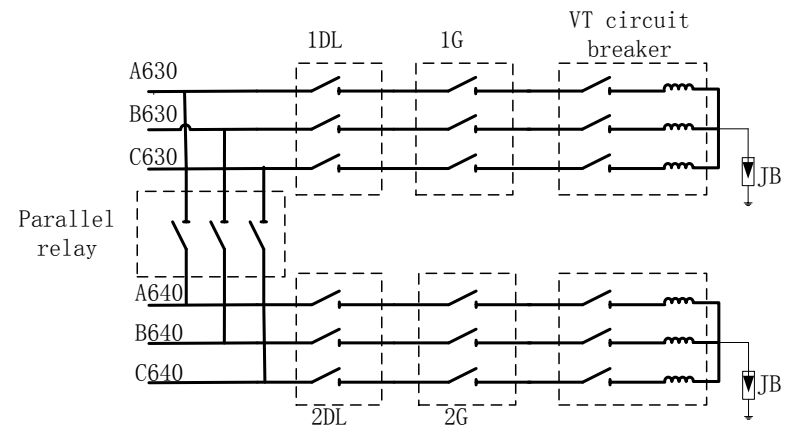

Figure 3. Improved voltage parallel diagram

The auxiliary contact of the incoming circuit breaker of the corresponding line is connected in series to the isolation switch of the connecting bus side. In two lines respectively in two buses running, two circuit breakers normally open contact closed, the voltage transformer normal operation voltage, thus voltage relay is closed, if the voltage relay is parallel, secondary circuit voltage parallel.

When the circuit breaker is tripped, the normally open contact of the circuit breaker is disconnected, even if the parallel relay is operated, the secondary voltage is in parallel, and the utility model can not cause the secondary voltage sending to the losing line voltage transformer. In this way, we can avoid the problem of the secondary circuit reverse transmission of the voltage transformer.

In the same time, this scheme is easier to be realized in the early design stage. 


\section{Conclusion}

According to the bridge connection line voltage transformer and special bus voltage transformer, analyzes the bridge backup automatic switch mode, line fault may cause the secondary circuit reverse transmission of the voltage transformer.

Three solutions are proposed for this kind of situation, the voltage transformer removing, the output port of backup automatic switch increasing, and increasing the contact position of the circuit breaker. The advantages and disadvantages of the three schemes are compared, which provides an effective reference for designers.

To sum up, according to a different connection scheme, there is a big difference between the two wiring circuit voltage juxtaposition device, in the design, we must carefully analyze a wiring scheme of voltage juxtaposition device, to ensure that the design is correct and reasonable.

\section{References}

[1] Yang, Yongbo, Y. Wang, and D. Su. "The Incorrect Action Analysis Of Two-stage Backup Automatic Switch." International Conference on Management, Computer and Education Informatization 2015.

[2] Yang, Yongbo, et al. "Analysis of the Failure of Backup Automatic Switch in Substation." International Conference on Electronic, Mechanical, Information and Management Society 2016.

[3] Sun Yahui, Chen Zhiqiang, Cai Yan. Reason analysis and preventive measures of the two circuit of the voltage transformer[J]. Power System Protection and Control, 2010, 38(9):126-129.

[4] Li Peng, Zhong Zhuhui. Development of a device for preventing the counter voltage mutual inductor[J]. Power System Protection and Control, 2014(13):135-138

[5] Zhang Yaohong, Yuan Feng, etc.The voltage criterion of PT secondary voltage and the auxiliary contact of the tool gate two[J]. Power System Protection and Control, 2011, 39(15):137-140.

[6] Q / GDW 426 - 2010Technical specification for merging unit of intelligent substation [M]. China Electric Power Press, 2010.

[7] Huang Changshu, $\mathrm{Hu}$ Yunhua. The problems and Countermeasures of device wiring in [J]. electric power automation equipment, 2009, 29(4):147-149.

[8] Zhang Bao-hui, Yin Xiang-gen.Power system protection [M]. China Electric Power Press, 2005.

[9] GB / T 584-2007, $3 \sim 110 \mathrm{kV}$ power grid protection devices running the tuning procedure [S].

[10] Shenzhen Nari Technology Co. Ltd.. ISA-358 standby power supply automatic switching device technology specification [EB / OL]. 